\title{
Altitude Sickness in Climbers and Efficacy of NSAIDs Trial (ASCENT): Randomized, Controlled Trial of Ibuprofen Versus Placebo for Prevention of Altitude Illness
}

Jeffrey H. Gertsch, MD; Bryan Corbett, MD; Peter S. Holck, PhD; Allison Mulcahy, MD; Melanie Watts, MD; Nathan Thomas Stillwagon, MD; Amanda Morgan Casto, MD; Charles Hessel Abramson, MD; Charles Peter Aloysius Vaughan, MD; Christopher Macguire, MD; Neda Nicole Farzan, MD; Baotran Nguyen Vo, MD; Rebecca Jean Norvelle, MD; Kerstin May, MD; Jessica Elizabeth Holly, MD; Hillary Irons, MD; Aaron Michael Stutz, MD; Pradip Chapagain, MD; Siddhartha Yadav, MD; Matiram Pun, MD; Jeremy Farrar, BSc, MBBS, FRCP, DPhil, OBE; Buddha Basnyat, MD, MSc, FRCP(E)

From the Department of Neurosciences, University of California-San Diego School of Medicine, San Diego, CA (Dr Gertsch); Himalayan Rescue Association, Kathmandu, Nepal (Drs Corbett, Mulcahy, Watts, Stillwagon, Casto, Abramson, Vaughan, Macguire, Farzan, Vo, Norvelle, May, Holly, Irons, Stutz, and Basnyat), Department of Public Health Sciences and Epidemiology, John A. Burns School of Medicine, University of Hawaii, Honolulu, HI (Dr Holck); Mountain Medicine Society of Nepal (MMSN), Nepal (Drs Chapagain, Yadav, Pun, and Basnyat); University of Calgary, Calgary, Canada (Dr Pun); Oxford University Clinical Research Unit-Vietnam, Hanoi, Vietnam (Dr Farrar); Nepal International Clinic (NIC), Nepal (Dr Basnyat); and Patan Academy of Health Sciences (PAHS), Nepal (Dr Basnyat).

Objective.-To study the effectiveness of ibuprofen versus placebo in preventing acute mountain sickness (AMS) and high altitude headache (HAH).

Methods.-Double-blind, randomized, placebo-controlled trial.

Results.-Two hundred ninety-four healthy Western trekkers were recruited on the Everest approach at $4280 \mathrm{~m}$ or $4358 \mathrm{~m}$ and randomly assigned to receive either $600 \mathrm{mg}$ of ibuprofen or placebo 3 times daily before and during ascent to $4928 \mathrm{~m}$. One hundred eighty-three of 294 participants completed the trial. Of the participants who did not complete the trial, 62 were lost to follow-up and another 49 broke trial protocol. In an intent-to-treat analysis (232 participants), ibuprofen was found to be more effective than placebo in reducing the incidence of AMS $(24.4 \%$ vs $40.4 \% ; P=.01)$ and the incidence of HAH (42.3\% vs $60.5 \% ; P<.01)$. Ibuprofen was also superior to placebo in reducing the severity of HAH $(4.9 \%$ vs $14.7 \% ; P=.01)$. The end point of oxygen saturation was also higher in the ibuprofen group ( $80.8 \%$ vs $82.4 \% ; P=.035)$. For the 183 participants who completed the trial and conformed to the protocol, the incidence of AMS between placebo and treatment groups was not significant $(32.9 \%$ vs $22.7 \% ; P=.129$ for AMS incidence, $9.6 \%$ vs $8.2 \% ; P=.74$ for AMS severity, $54.8 \%$ vs $42.7 \% ; P=$ .11 for HAH incidence, and $8.2 \%$ vs $3.6 \% ; P=.18$ for HAH severity).

Conclusions.-Ibuprofen was found to be effective in preventing AMS in the intent-to-treat analysis group but not in those who completed the trial. This loss of significance in the subjects who completed the trial may be explained by persons in the placebo group having a higher burden of illness and associated decreased compliance with the protocol. An important limitation of this study may be the possibility that ibuprofen can mask headache, which is a compulsory criterion for the diagnosis of AMS.

Key words: altitude, acute mountain sickness, Nepal, high altitude headache, ibuprofen, Lake Louise Questionnaire, randomized controlled trial

Corresponding author: Buddha Basnyat, MD, MSc, FRCP(E), Himalayan Rescue Association, Kathmandu, Nepal, C/O Nepal International Clinic (NIC), GPO BOX 3596, Lal Durbar, Kathmandu, Nepal ( e-mail: rishibas@wlink.com.np).

\section{Introduction}

Acute mountain sickness (AMS) is a disease process associated with hypobaric hypoxemia and defined by a group of symptoms occurring at altitudes greater than $2500 \mathrm{~m}$ as defined by the 1991 Lake Louise Consensus 
Group. ${ }^{1}$ These symptoms include gastrointestinal upset, sleep disturbances, weakness/fatigue, dizziness/lightheadedness, and most importantly high altitude headache (HAH) - the requisite symptom for the diagnosis of AMS. Both AMS and HAH commonly occur within 6 to 24 hours of ascent to altitudes greater than $2500 \mathrm{~m}$ with substantial morbidity with increasing altitude $(42 \%$ at $3000 \mathrm{~m}$ for AMS and $47 \%-68 \%$ at $4928 \mathrm{~m}$ for HAH). ${ }^{1-5}$ Although AMS is often a self-limiting and benign process, if untreated it can progress to high altitude cerebral edema (HACE), a condition characterized by ataxia, altered level of consciousness, vasogenic cerebral edema, coma, and death. ${ }^{1,6}$ The potential lethality of HACE underscores the importance of effective prevention of AMS and $\mathrm{HAH}$.

Although the mainstay of AMS prevention is a gradual ascent, acetazolamide has been used as the standard prophylactic medicine for decades. ${ }^{7}$ In addition to acetazolamide, nonsteroidal anti-inflammatory drugs (NSAIDs) such as aspirin and more recently ibuprofen have been shown to be effective in the prevention of $\mathrm{HAH}^{8-11}$ In a previous study, ${ }^{11}$ our research group found ibuprofen to be similar to acetazolamide in preventing HAH and AMS. These results, taken together with other data suggestive of an inflammatory component to hypobaric hypoxemic pathology, suggested that an NSAID such as ibuprofen could counter AMS. For this reason, we designed a field-based trial to verify and extend our previous clinical findings in the Himalaya.

\section{Methods}

This study was designed as a prospective, double-blind, randomized, placebo-controlled, field-based clinical trial. Enrollment took place between October and November 2009 along the approach trail to Mount Everest in the Nepal Himalaya. This study was conducted in accordance with the Declaration of Helsinki; the Oxford Tropical Research Ethics Committee and the Nepal Health Research Council provided institutional review. The trial was duly registered (ISRCTN 91790322).

\section{PARTICIPANTS AND OUTCOME MEASURES}

Trekkers completed questionnaires after giving signed informed consent. Inclusion criteria specified healthy non-Nepali males and females 18 to 65 years of age traveling directly between the baseline villages of Pheriche or Dingboche (4280 m and $4358 \mathrm{~m}$, respectively) and the end point in Lobuje (4928 m). Potential participants were excluded if they had any more than 2 mild symptoms $(\leq 1)$ on the Lake Louise AMS Questionnaire (LLQ), signs or symptoms of a substantial acute infection, had slept above $4500 \mathrm{~m}$, or had taken any NSAIDs or acetazolamide within 1 day or 3 days before enrollment, respectively.

The predetermined primary outcome measure was AMS incidence at the study end point as calculated on the LLQ, a validated field standard for the diagnosis of AMS. ${ }^{12}$ Predetermined secondary end points included evaluation of AMS severity, as well as HAH incidence and severity on the LLQ and visual analog scale (VAS). Other secondary measures included pulse oximetry $\left(\mathrm{SpO}_{2}\right)$ readings (Nonin Medical Products, Minneapolis, MN). Data concerning demographics, ascent profile, compliance, and side effects were collected to adjust for potential confounders.

\section{STUDY DESIGN}

Commercial pharmaceutical-grade ibuprofen and placebo were packed in visually identical capsules by Deurali-Janta Pharmaceuticals (Kathmandu, Nepal). Study medications were randomized via computer-generated code. Participants were sought out on a daily basis in all baseline village hotels and sequentially enrolled to minimize selection bias.

Newly arrived trekkers were recruited daily to participate, and volunteers meeting inclusion criteria were interviewed to obtain data. Interviewers gathered demographics, ascent profile data, LLQ, VAS, and $\mathrm{SpO}_{2}$ data points. All trekkers were given information on methods for reducing the risk of AMS and other forms of altitude illness, thereby meeting the minimum standard of care. Participants were then randomly assigned (in a doubleblind fashion) to ingest visually indistinguishable capsules 3 times daily of either placebo or ibuprofen 600 mg. Participants took a minimum of 3 doses at the baseline altitude before proceeding on their trek.

On their ascent from baseline, a minority of participants stopped overnight at a lodge at $4595 \mathrm{~m}$, but all were expected to arrive at the end point altitude for data collection. The VAS and LLQ scores were self-reported the night of arrival and the morning after arrival, at which point the study was complete. End point data collection represents morning-after-arrival data (unless missing, then replaced with night-before data) to emphasize specificity in the diagnosis of HAH and AMS.

Study administrators at baseline and end point sites were trained to be vigilant for severe illness that might be attributable to altitude or a reaction to medications, equipped with appropriate rescue medications and $\mathrm{Ga}$ mow bag (portable hyperbaric chamber), and always ready to help with descent. 


\section{STATISTICAL ANALYSIS}

Power calculations suggested a minimum total of 164 participants ( 82 per arm) was needed, a total calculated to be sufficient to identify a statistically significant difference (at $80 \%$ power) between treatment and placebo based on published HAH incidence of $50 \%$ and AMS incidence of $32 \%$ at the study end point. ${ }^{11}$ Intent-to-treat analyses, univariate comparisons (Pearson $\chi^{2}$, Fisher exact tests, $t$ tests), and logistic regression were used to examine multivariate risk factors for binary outcomes. Probability values less than 0.05 were considered significant. All analyses were conducted using R 2.6.0 software (R Foundation for Statistical Computing, Vienna, Austria).

\section{Results}

Two hundred ninety-four total participants were enrolled in the study, with 183 completing the entirety of the protocol (Figure). Forty-nine participants broke protocol for various reasons, but did present at the completion of the study to allow for data acquisition. The remaining 62 participants (26.7\%) were lost to follow-up; an expected finding consistent with previous studies. ${ }^{2,4,11,13}$ An intent-to-treat analysis per protocol was used to analyze all accumulated data. Analysis of demographic data revealed no statistically significant differences between the ibuprofen and placebo groups with respect to gender, age, nights spent at altitude, mode of arrival to the Himalayas, LLQ score at baseline, previous use of medication for altitude illness, $\mathrm{SpO}_{2}$, VAS, or altitude of residence.

With regard to adverse events, one participant among the 232 reported black stool and was advised to stop the study medication (the code was broken and he was found to be on ibuprofen). He was followed closely with timely physical examinations with no untoward event. Ten other persons reported stomach pain, 3 in the ibuprofen group and 7 in the placebo group. For all, the pain subsided within a day with no additional medication. There were no patients with HACE or HAPE.

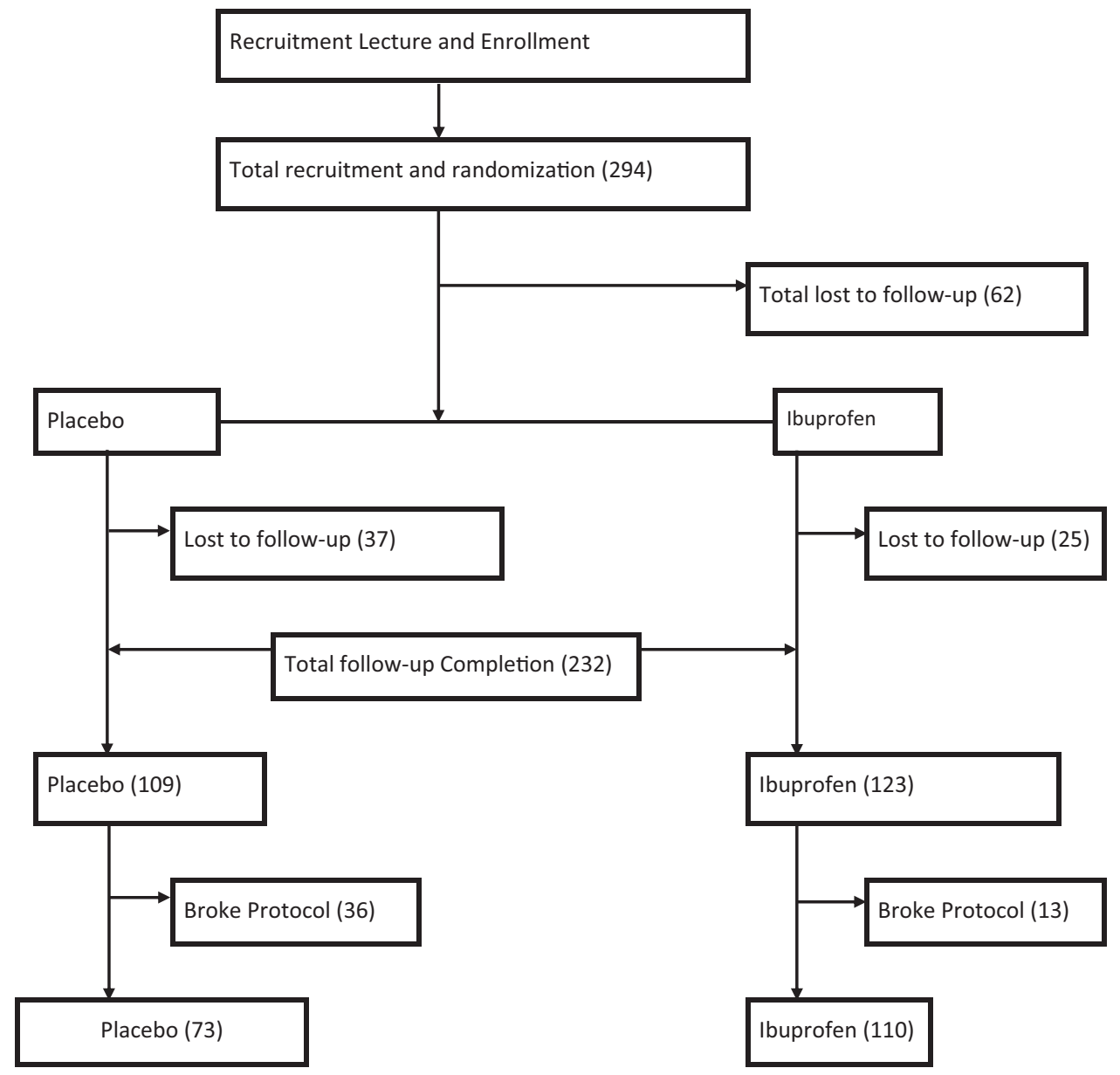

Figure. Participant flow diagram. 
Table 1. Baseline characteristics of the intent-to-treat group

\begin{tabular}{|c|c|c|c|c|c|c|c|}
\hline \multirow[b]{2}{*}{ Variables } & \multicolumn{2}{|c|}{ Study participants } & \multicolumn{2}{|c|}{ Placebo group } & \multicolumn{2}{|c|}{ Ibuprofen group } & \multirow[b]{2}{*}{$P$ value } \\
\hline & $N$ & $\%$ & $N$ & $\%$ & $N$ & $\%$ & \\
\hline Cohort at end point (of 294) ${ }^{a}$ & 232 & N/A & 109 & $47.0 \%$ & 123 & $53.0 \%$ & N/A \\
\hline Female gender & 81 & $35.2 \%$ & 35 & $32.4 \%$ & 46 & $37.7 \%$ & .401 \\
\hline Mean age (y) & $37 \pm 12$ & N/A & $36 \pm 11$ & N/A & $38 \pm 12$ & N/A & .353 \\
\hline Trekkers starting from $2800 \mathrm{~m}^{b}$ & 196 & $86.3 \%$ & 93 & $86.9 \%$ & 103 & $85.8 \%$ & .813 \\
\hline Nights to ascend to baseline & $3.4 \pm 0.8$ & N/A & $3.4 \pm 0.9$ & N/A & $3.5 \pm 0.7$ & N/A & .289 \\
\hline Enrolled at $4358 \mathrm{~m}^{c}$ & 143 & $61.9 \%$ & 71 & $65.1 \%$ & 72 & $59.0 \%$ & .339 \\
\hline History of altitude illness & 12 & $5.3 \%$ & 5 & $4.7 \%$ & 7 & $5.8 \%$ & .720 \\
\hline Positive LLQ score ${ }^{d}$ & 129 & $55.6 \%$ & 62 & $56.9 \%$ & 67 & $54.5 \%$ & .712 \\
\hline LLQ headache score $=1$ & 107 & $46.1 \%$ & 51 & $46.8 \%$ & 56 & $45.5 \%$ & .848 \\
\hline Enrollment $\mathrm{SpO}_{2}$ & $85.9 \pm 6.3$ & N/A & $85.2 \pm 8.4$ & N/A & $86.5 \pm 3.4$ & N/A & .287 \\
\hline Headache VAS & $0.5 \pm 0.9$ & N/A & $0.6 \pm 1.0$ & N/A & $0.4 \pm 0.8$ & N/A & .506 \\
\hline Lost to follow-up & 62 & $26.7 \%$ & 37 & $59.7 \%$ & 25 & $40.3 \%$ & .40 \\
\hline
\end{tabular}

AMS, acute mountain sickness; LLQ, Lake Louise AMS Questionnaire; N/A, not applicable; $\mathrm{SpO}_{2}$, oxygen saturation by pulse oximetry; VAS, visual analog scale.

${ }^{a}$ End point cohort consists of participants enrolled at baseline and followed up at end point $(n=294)$.

${ }^{b}$ The Lukla airport is at $\sim 2800 \mathrm{~m}$, and trekkers starting from Jiri $(2000 \mathrm{~m})$ pass through Lukla.

${ }^{c}$ Enrollment occurred in the villages of Dingboche $(4358 \mathrm{~m})$ and Pheriche $(4280 \mathrm{~m})$.

${ }^{d}$ Participants were allowed to have one mild (LLQ $\left.=1\right)$ nonheadache symptom on the Lake Louise questionnaire to prevent self-selection bias.

\section{INTENT-TO-TREAT ANALYSIS}

Table 1 reveals the baseline characteristics of the intentto-treat group, and the main outcome profile of that group is presented in Table 2. (The intent-to-treat group comprises the group based on initial treatment intent, not on treatment eventually administered). Several statistically significant results were observed for the ibuprofen group as compared with placebo, including a decreased incidence of HAH $(42.3 \%$ vs $60.5 \% ; P<.01)$ and AMS $(24.4 \%$ vs $40.4 \% ; P<.01)$, as well as a decrease in severity of HAH on the LLQ and VAS (HAH $>1$ on LLQ, $4.9 \%$ vs $14.7 \% ; P=.01$; VAS, $0.7 \pm 1.5$ vs $1.1 \pm$ $1.7 ; P=.04$ ). Additionally, end point $\mathrm{Spo}_{2}$ was greater in the ibuprofen group vs the placebo group $(82.8 \% \pm$

Table 2. Main outcome profile (intent-to-treat)

\begin{tabular}{|c|c|c|c|c|c|c|c|}
\hline \multirow[b]{2}{*}{ Variables } & \multicolumn{2}{|c|}{ Study participants } & \multicolumn{2}{|c|}{ Placebo group } & \multicolumn{2}{|c|}{ Ibuprofen group } & \multirow[b]{2}{*}{$P$ value } \\
\hline & $N$ & $\%$ & $N$ & $\%$ & $N$ & $\%$ & \\
\hline End point cohort (of 294) & 232 & N/A & 109 & $47.0 \%$ & 123 & $53.0 \%$ & N/A \\
\hline Headache incidence & 118 & $50.9 \%$ & 66 & $60.5 \%$ & 52 & $42.3 \%$ & 0.005 \\
\hline Severe headache incidence ${ }^{b}$ & 22 & $9.5 \%$ & 16 & $14.7 \%$ & 6 & $4.9 \%$ & 0.011 \\
\hline AMS incidence $^{c}$ & 74 & $31.9 \%$ & 44 & $40.4 \%$ & 30 & $24.4 \%$ & 0.009 \\
\hline Severe AMS incidence ${ }^{d}$ & 24 & $10.3 \%$ & 13 & $11.9 \%$ & 11 & $8.9 \%$ & 0.456 \\
\hline End point $\mathrm{SpO}_{2}(\%)$ & $81.6 \pm 4.9$ & N/A & $80.8 \pm 4.7$ & N/A & $82.4 \pm 5.0$ & N/A & 0.035 \\
\hline $\mathrm{SpO}_{2}$ decrease from baseline & $4.1 \pm 7.8$ & N/A & $4.1 \pm 10$ & N/A & $4.2 \pm 5$ & N/A & 0.157 \\
\hline Headache visual analog scale (VAS) & $0.9 \pm 1.6$ & N/A & $1.1 \pm 1.7$ & N/A & $0.7 \pm 1.5$ & N/A & 0.043 \\
\hline Participants who broke protocol ${ }^{e}$ & 49 & $21.1 \%$ & 36 & $33.0 \%$ & 13 & $10.6 \%$ & 0.01 \\
\hline
\end{tabular}

AMS, acute mountain sickness; N/A, not applicable; $\mathrm{SpO}_{2}$, oxygen saturation by pulse oximetry.

${ }^{a}$ Probability value for comparison between placebo and ibuprofen groups.

${ }^{b}$ Severe headache incidence as determined by a value of 2 or greater (of 0-3) on the Lake Louise AMS Questionnaire, "do you have a headache?"

${ }^{c}$ AMS defined as Lake Louise AMS Questionnaire score of 3 or greater, requiring headache plus one or more symptoms.

${ }^{d}$ Severe AMS incidence as determined by a Lake Louise AMS Questionnaire score of 5 or greater.

${ }^{e}$ Participants who broke protocol were defined as those taking a medication conflicting with their assigned treatment group (crossovers) or those who missed 2 or more doses. 
Table 3. Main outcome profile excluding those who broke protocol ${ }^{a}$

\begin{tabular}{|c|c|c|c|c|c|c|c|}
\hline \multirow[b]{2}{*}{ Variables } & \multicolumn{2}{|c|}{ Study participants } & \multicolumn{2}{|c|}{ Placebo group } & \multicolumn{2}{|c|}{ Ibuprofen group } & \multirow[b]{2}{*}{$P$ value $^{b}$} \\
\hline & $N$ & $\%$ & $N$ & $\%$ & $N$ & $\%$ & \\
\hline End point cohort (of 294) & 183 & N/A & 73 & $40.0 \%$ & 110 & $60.0 \%$ & N/A \\
\hline Headache incidence & 87 & $47.5 \%$ & 40 & $54.8 \%$ & 47 & $42.7 \%$ & 0.109 \\
\hline Severe headache incidence ${ }^{c}$ & 10 & $5.5 \%$ & 6 & $8.2 \%$ & 4 & $3.6 \%$ & 0.182 \\
\hline AMS incidence ${ }^{d}$ & 49 & $26.8 \%$ & 24 & $32.9 \%$ & 25 & $22.7 \%$ & 0.129 \\
\hline Severe AMS incidence ${ }^{e}$ & 16 & $8.7 \%$ & 7 & $9.6 \%$ & 9 & $8.2 \%$ & 0.741 \\
\hline End point $\mathrm{SpO}_{2}(\%)$ & $82.2 \pm 4.7$ & N/A & $81.4 \pm 4.4$ & N/A & $82.7 \pm 4.8$ & N/A & 0.065 \\
\hline $\mathrm{SpO}_{2}$ decrease from baseline & $3.9 \pm 8.5$ & N/A & $3.8 \pm 11.7$ & N/A & $4.0 \pm 5$ & N/A & 0.1 \\
\hline $\begin{array}{l}\text { Headache visual analog } \\
\text { scale (VAS) }\end{array}$ & $0.6 \pm 1.3$ & N/A & $0.6 \pm 1.1$ & N/A & $0.6 \pm 1.3$ & N/A & 0.458 \\
\hline
\end{tabular}

AMS, acute mountain sickness; N/A, not applicable; $\mathrm{SpO}_{2}$, oxygen saturation by pulse oximetry.

${ }^{a}$ Participants who broke protocol were defined as those taking a medication conflicting with their treatment group (crossovers) or those who missed 2 or more doses.

${ }^{b}$ Probability value for comparison between placebo and ibuprofen groups.

${ }^{c}$ Severe headache incidence as determined by a value of 2 or greater (of $0-3$ ) on the Lake Louise AMS Questionnaire; "do you have a headache?"

${ }^{d}$ AMS defined as Lake Louise AMS Questionnaire score of 3 or greater, requiring headache plus one or more symptoms.

${ }^{e}$ Severe AMS incidence as determined by a Lake Louise AMS Questionnaire score of 5 or greater.

$5.0 \%$ vs $80.8 \% \pm 4.7 \% ; P=.03)$. No statistically significant differences were observed between treatment groups in AMS severity.

\section{ANALYSIS OF FULLY COMPLIANT PARTICIPANT DATA}

Table 3 shows the main outcome profile of the fully compliant participants. Analysis among fully compliant participants (those who hiked from the baseline to end point, missed fewer than 2 doses of assigned medication, and abstained from using nonstudy medications) found no statistically significant differences in any outcomes measured. Although not statistically significant, the data did demonstrate a difference between ibuprofen and placebo groups with respect to $\mathrm{HAH}$ and AMS incidence and severity, consistent with results of the intent-to-treat analysis $(42.7 \%$ vs $54.8 \%$ for HAH incidence; $3.64 \%$ vs $8.2 \%$ for HAH score $>$ $1 ; 22.7 \%$ vs $32.9 \%$ for AMS incidence; $8.2 \%$ vs $9.6 \%$ for AMS score $>5$ on LLQ).

\section{MULTIVARIATE ANALYSIS}

Multivariate models were developed for 6 different outcomes: headache incidence; severe headache incidence; AMS incidence; severe AMS incidence; end point VAS; and $\mathrm{SpO}_{2}$ change. Covariates included in these models (in addition to treatment status) were "broke protocol" group, mode of arrival, nights spent, enrolled at Ding- boche, gender, age, and medications taken previously for altitude illness.

Statistically significant differences in outcome between treatment groups were found for headache incidence $(P=.01)$, and indications of differences were observed for AMS incidence $(P=.07)$.

\section{ANALYSIS OF PARTICIPANTS WHO BROKE PROTOCOL}

Analysis of the participants breaking protocol compared with fully compliant participants revealed a higher incidence of AMS (51\% vs $26.8 \% ; P=.001)$ and incidence of more severe HAH scores based on both LLQ and VAS (HAH score $>1$ on LLQ $24.5 \%$ vs $5.5 \% ; P<.01$; VAS $1.75 \pm 2.33$ vs $0.62 \pm 1.26 ; P<.01)$. Although not quite a statistically significant difference, participants who broke protocol had an increased incidence of $\mathrm{HAH}$ $(63.3 \%$ vs $47.5 \% ; P=.051)$ compared with those who were fully compliant. No statistically significant difference was observed in $\mathrm{SpO}_{2}$ change from baseline (4.95 \pm 4.4 vs $3.9 \pm 8.52 ; P=.59$ ) or AMS severity (AMS score $>516.3 \%$ vs $8.7 \% ; P=.12$ ) when comparing participants who broke protocol with compliant participants (although both scores were better among compliant participants). Persons who broke protocol were significantly more likely to be in the placebo group than the ibuprofen group $(P<.01)$. Headache was the most common reason reported for breaking protocol, with placebo group members more frequently reporting this reason as compared 
with the treatment group (other reasons for taking additional medications included muscle pain and a catchall category termed "other").

\section{LOST TO FOLLOW-UP ANALYSIS}

Analysis of those who completed the study versus those lost to follow-up revealed 2 measured variables for which statistically significant differences between the 2 groups were observed; nights spent at altitude (3.42 \pm 0.778 vs $4.37 \pm 1.42 ; P<.01)$ and baseline $\mathrm{Spo}_{2}(85.9 \%$ vs $84.9 \% ; P<.01)$. Other demographics including age, gender, mode of arrival, baseline LLQ score reading, and resident altitude showed no significant variation between those who completed the study and those lost to followup. Finally, $16.9 \%$ of the treatment group was lost to follow-up, compared with $25.3 \%$ of the placebo group $(P=.10)$.

\section{EXCLUSION ANALYSIS}

Analysis of persons enrolled in the study vs persons excluded revealed only 1 variable with a statistically significant difference between the groups: age (36.5 \pm 11.1 vs $31.6 \pm 10.6 ; P=.011)$. There were no significant differences between groups with respect to gender, mode of arrival, nights spent at altitude, or oxygen saturation at baseline.

\section{Discussion}

The present study represents an attempt to verify and extend observations from our previous trial. ${ }^{11}$ Many of the findings were reconfirmed here in an intent-to-treat analysis (Table 2); ibuprofen again showed a significant clinical reduction in the incidence (but not severity) of AMS and HAH. However, for the participants who completed the trial without breaking protocol (Table 3), estimated differences in the incidence and severity of both HAH and AMS in the ibuprofen group vs the placebo group dropped below the threshold necessary for statistical significance. We hypothesize that the loss of statistical significance observed in some outcome measures when persons breaking protocol were excluded stems from the exclusion of data from the sickest participants. The data in Table 2 demonstrate that participants in the placebo group bore a greater burden of illness, and thus would be expected to have a higher rate of noncompliance resulting in exclusion from the data set in Table 3 (placebo $[n=36]$ vs ibuprofen $[n=13] ; P=.01$; odds ratio, 4.2). Thus, we recommend that the loss of statistical significance shown in Table 3 should be interpreted with caution. Indeed, both AMS incidence and HAH severity were statistically significantly higher in the cohort that broke protocol. Those who broke protocol also had a higher HAH incidence than those continuing on in the study $(P=.051)$.

We were surprised to find that many outcomes that were significantly different between treatment groups in univariate analyses were no longer significant in our multivariate analyses. Although some loss of precision in estimation is to be expected with the loss of degrees of freedom accompanying increased terms in a model, we posit that the inclusion of a covariate specifying "broke protocol" in our multivariate models also contributed to the "washing out" of significant differences. The colinearity of treatment status and "broke protocol" status results in larger standard errors for each of these coefficient estimates. Indeed, when the "broke protocol" covariate was removed from the multivariate models, outcomes of AMS and HAH incidence were again statistically significant (data not shown).

Finally, although not statistically significant $(P=.10)$, persons taking placebo were estimated to be somewhat more likely to be lost to follow-up than were persons in the ibuprofen group, as was the case noted above with breaking protocol. We suggest our observed results in the fully compliant analyses may be partially biased, whereby treatment appears less effective than it actually is. The less compliant participants who broke protocol were likely more affected by the altitude. This group was also more likely to be those individuals taking placebo than those taking ibuprofen. Thus, analyses of fully compliant participants, including adjustments for breaking protocol, contribute to a diminished observed treatment effect among the fully compliant.

Previous HAH prevention studies with aspirin demonstrated a significant protective effect. ${ }^{8,9}$ This is consistent with the present study, which demonstrated a reduction in the incidence and severity of HAH in the intent-totreat analysis. Similarly, Meehan et al ${ }^{14}$ studied naproxen for prevention of AMS; however, they found no statistically significant differences to suggest a protective effect. These results should be viewed with caution, as the study likely lacked sufficient power to come to any strong conclusion with a sample size of just 11 participants. As we were submitting this article, another study ${ }^{15}$ carried out at a lower altitude $(3810 \mathrm{~m})$ with a smaller sample size (86 participants) than this present study was published. The study, which was conducted in the White Mountains of California, showed ibuprofen to be efficacious in the prevention of AMS.

Our study was inconsistent with the results of Kayser et $\mathrm{al}^{16}$ looking at the effects of calcium carbasalate for prevention of AMS and HAH. They found no evidence 
of calcium carbasalate having a protective effect for AMS or HAH; however, the study also suffered from significant methodologic problems with blinding and an inadequate sample size. ${ }^{17}$

Roach et $\mathrm{al}^{18}$ demonstrated elevated levels of antiinflammatory markers in individuals less susceptible to AMS. The protective effects of anti-inflammatory markers point toward inflammation as important in the pathophysiology of AMS. There has been recent interest in the concept that inflammation may contribute to the development of cerebral forms of altitude illness. Vasogenic cerebral edema is known to occur secondary to exposure to hypobaric hypoxemia and increased cerebral blood flow. ${ }^{19,20}$ It is also widely recognized that the generation of this vasogenic cerebral edema requires some disruption of the blood-brain barrier. ${ }^{21}$ In addition to such edema, hypobaric hypoxemia has been shown to result in elevated levels of inflammatory markers and anti-inflammatory markers in those susceptible and resistant to altitude illness, respectively. ${ }^{18,22,23}$ Although the causal relationship of these findings remains unclear, their association has led to the development of the theory that hypobaric hypoxemia-induced inflammation may play a role in blood-brain barrier breakdown, which is one important step in the development of AMS and subsequent HACE. Ibuprofen may inhibit this blood-brain barrier breakdown.

The etiology of the pain of HAH also continues to be hypothetical. What is known is that irritation of the meningovascular system by various mechanisms is important in the development of various forms of headaches. ${ }^{6,7,22,24-26}$ Traction on the meningovascular system as a consequence of brain swelling and increased cerebral blood flow has been proposed as a possible mechanism for the development of the characteristic HAH of AMS. ${ }^{19,20}$ Chemical irritation or sensitization of this system is also thought to take place. For example, inflammatory mediators such as prostaglandins are known to potentiate nociception and thus magnify the mechanical irritation described above. ${ }^{23}$ The possible role of inflammation in the development of HAH is another pathway by which anti-inflammatory drugs such as NSAIDs may prevent $\mathrm{HAH}^{27-30}$

\section{LIMITATIONS OF THE STUDY}

There are several limitations to the application of these data in clinical practice. This cohort we studied at high altitude is partially acclimatized even though the end point data were collected at about $700 \mathrm{~m}$ higher than the study enrollment altitude. We have used this site for a number of randomized, controlled trials in the past, ${ }^{2,3,11}$ whose results have been verified by other studies from other venues. ${ }^{31}$ It is well known that acetazolamide can be taken episodically to hasten acclimatization even while trekkers are ascending. We reasoned that another drug that performed likewise would be a useful alternative. Importantly, adequately powered high altitude studies are scarce, and this site gives us an opportunity to study large numbers of participants. Second, because just over a fifth of enrollees were lost to follow-up, our results may be biased, particularly when being lost to follow-up may be directly related to AMS status. Analysis of those lost to follow-up suggests participants in the placebo group were more likely to drop out of the study. Attrition of this magnitude is expected as in our previous studies in the past in this region, ${ }^{2,4}$ given the realities of conducting a study in a wilderness setting. Third, we observed a statistically significant difference between the age of participants and the age of persons excluded from the trial (who on average were about 5 years younger). More advanced age is known to have a protective effect on brain volume, and this difference could conceivably result in a lower AMS incidence and severity bias. Fourth, the mean baseline oxygen saturation of the group lost to follow-up was significantly lower than that of persons who completed the study. This difference too may have led to lower AMS incidence bias in the cohort, ${ }^{32}$ although it is difficult to know whether a median difference of $1 \%$ between the 2 groups in baseline oxygen saturation is clinically significant, albeit statistically significant. Finally, an important limitation of the study is that ibuprofen is effective in prevention and treatment of headache, which is not only the most prevalent symptom of AMS but a compulsory criterion for the diagnosis of AMS. ${ }^{33,34}$ Hence, it may be problematic to distinguish the pure analgesic effect from a treatment effect of AMS.

\section{Conclusions}

Until basic research and more studies confirm the mechanism behind AMS using ibuprofen, we urge caution when using ibuprofen and other NSAIDs for the prevention of HAH and AMS. The clinical results we observed suggest ibuprofen is an inexpensive, over-the-counter prophylactic agent. However, an alternative, arguable conclusion is that ibuprofen merely masks a useful marker (headache) of AMS. Further study is warranted.

\section{Acknowledgments}

Funding for this study was provided by Wellcome Trust, UK. We would like to thank all the staff of the Himalayan Rescue Association in Nepal and members of Moun- 
tain Medicine Society of Nepal (MMSN) who helped us tremendously to carry out this study. We are especially indebted to the trekker participants who were very kind to enroll in our study.

\section{References}

1. Hackett PH, Roach RC. High-altitude illness. N Engl J Med. 2001;345:107-114.

2. Basnyat B, Gertsch JH, Holck PS, et al. Acetazolamide 125 $\mathrm{mg} \mathrm{BD}$ is not significantly different from $375 \mathrm{mg} \mathrm{BD}$ in the prevention of acute mountain sickness: the prophylactic acetazolamide dosage comparison for efficacy (PACE) trial. High Alt Med Biol. 2006;7:17-27.

3. Basnyat B, Murdoch DR. High-altitude illness. Lancet. 2003;361:1967-1974.

4. Gertsch JH, Basnyat B, Johnson EW, Onopa J, Holck PS. Randomised, double blind, placebo controlled comparison of ginkgo biloba and acetazolamide for prevention of acute mountain sickness among Himalayan trekkers: the prevention of high altitude illness trial (PHAIT). BMJ. 2004;328: 797.

5. Honigman B, Theis MK, Koziol-McLain J, et al. Acute mountain sickness in a general tourist population at moderate altitudes. Ann Intern Med. 1993;118:587-592.

6. Hackett PH, Roach RC. High altitude cerebral edema. High Alt Med Biol. 2004;5:136-146.

7. Dumont L, Mardirosoff C, Tramér MR. Efficacy and harm of pharmacological prevention of acute mountain sickness: quantitative systematic review. BMJ. 2000;321:267-272.

8. Burtscher M, Likar R, Nachbauer W, Philadelphy M. Aspirin for prophylaxis against headache at high altitudes: randomised, double blind, placebo controlled trial. $B M J$. 1998;316:1057-1058.

9. Burtscher M, Likar R, Nachbauer W, Philadelphy M, Pühringer R, Lämmle $\mathrm{T}$. Effects of aspirin during exercise on the incidence of high-altitude headache: a randomized, double-blind, placebo-controlled trial. Headache. 2001;41: 542-545.

10. Burtscher M, Philadelphy M, Likar R. Aspirin versus diamox plus aspirin for headache prevention during physical activity at high altitude. Adv Exp Med Biol. 1999;474: 370-371.

11. Gertsch JH, Lipman GS, Holck PS, et al. Prospective, double-blind, randomized, placebo-controlled comparison of acetazolamide versus ibuprofen for prophylaxis against high altitude headache: the Headache Evaluation at Altitude Trial (HEAT). Wilderness Environ Med. 2010;21: 236-243.

12. Roach RC, Bartsch P, Hackett P, Oelz O. The Lake Louise acute mountain sickness scoring system. In: Sutton J, Coates G, Huston C, eds. Hypoxia and Molecular Medicine: Proceedings of the 8th International Hypoxia Symposium, 9-13 February 1993; Lake Louise, Alberta, Canada. Burlington, VT: Queen City Printers; 1993. 272274.
13. Basnyat B, Hargrove J, Holck PS, et al. Acetazolamide fails to decrease pulmonary artery pressure at high altitude in partially acclimatized humans. High Alt Med Biol. 2008; 9:209-216.

14. Meehan RT, Cymerman A, Rock P, et al. The effect of naproxen on acute mountain sickness and vascular responses to hypoxia. Am J Med Sci. 1986;292:15-20.

15. Lipman GS, Kanaan NC, Holck PS, Constance BB, Gertsch JH; PAINS Group. Ibuprofen prevents altitude illness: a randomized controlled trial for prevention of altitude illness with nonsteroidal anti-inflammatories. Ann Emerg Med. 2012;59:484-490.

16. Kayser B, Hulsebosch R, Bosch F. Low-dose acetylsalicylic acid analog and acetazolamide for prevention of acute mountain sickness. High Alt Med Biol. 2008;9:15-23.

17. Basnyat B, Gertsch JH, Holck PC. Low-dose acetylsalicylic acid analog and acetazolamide for prevention of acute mountain sickness [letter]. High Alt Med Biol. 2008;9:349, $351-35-2$.

18. Julian CG, Subudhi AW, Wilson MJ, Dimmen AC, Pecha $\mathrm{T}$, Roach RC. Acute mountain sickness, inflammation, and permeability: new insights from a blood biomarker study. J Appl Physiol. 2011;111:392-399.

19. Ter Minassian A, Beydon L, Ursino M, Gardette B, Gortan C, Richalet JP. Doppler study of middle cerebral artery blood flow velocity and cerebral autoregulation during a simulated ascent of Mount Everest. Wilderness Environl Med. 2001;12:175-183.

20. Severinghaus JW, Chiodi H, Eger EI 2nd, Brandstater B, Hornbein TF. Cerebral blood flow in man at high altitude. Role of cerebrospinal fluid $\mathrm{pH}$ in normalization of flow in chronic hypocapnia. Circ Res. 1966;19:274-282.

21. Kallenberg K, Dehnert C, Dörfler A, et al. Microhemorrhages in nonfatal high-altitude cerebral edema. $J$ Cereb Blood Flow Metab. 2008;28:1635-1642.

22. Hartmann G, Tschöp M, Fischer R, et al. High altitude increases circulating interleukin-6, interleukin-1 receptor antagonist and C-reactive protein. Cytokine. 2000;12: 246-252.

23. Richalet JP, Hornych A, Rathat C, Aumont J, Larmignat P, Rémy P. Plasma prostaglandins, leukotrienes and thromboxane in acute high altitude hypoxia. Respir Physiol. 1991;85:205-215.

24. Levy D, Zhang XC, Jakubowski M, Burstein R. Sensitization of meningeal nociceptors: inhibition by naproxen. Eur J Neurosci. 2008;27:917-922.

25. Montgomery AB, Luce JM, Michael P, Mills J. Effects of dexamethasone on the incidence of acute mountain sickness at two intermediate altitudes. JAMA. 1989;261:734736.

26. Strassman AM, Raymond SA, Burstein R. Sensitization of meningeal sensory neurons and the origin of headaches. Nature. 1996;384:560-564.

27. Broome JR, Stoneham MD, Beeley JM, Milledge JS, Hughes AS. High altitude headache: treatment with ibuprofen. Aviat Space Environ Med. 1994;65:19-20. 
28. Harris NS, Wenzel RP, Thomas SH. High altitude headache: efficacy of acetaminophen vs. ibuprofen in a randomized, controlled trial. J Emerg Med. 2003;24:383387.

29. Hersh EV, Moore PA, Ross GL. Over-the-counter analgesics and antipyretics: a critical assessment. Clin Ther. 2000;22:500-548.

30. Sanchez del Rio M, Moskowitz MA. High altitude headache. Lessons from headaches at sea level. Adv Exp Med Biol. 1999;474:145-153.

31. Ritchie ND, Baggott AV, Todd WTA. Acetazolamide for the prevention of acute mountain sickness - a systematic review and meta-analysis. J Travel Med. 2012 July 30 [Epub ahead of print].

32. Roach RC, Greene ER, Schoene RB, Hackett PH. Arterial oxygen saturation for prediction of acute mountain sickness. Aviat Space Environ Med. 1998;69:1182-1185.

33. Roach R, Kayser B, Hackett P. Pro: headache should be a required symptom for the diagnosis of acute mountain sickness. High Alt Med Biol. 2011;12:21-22; discussion 29.

34. West JB. Con: headache should not be a required symptom for the diagnosis of acute mountain sickness. High Alt Med Biol. 2011;12:23-25; discussion 27. 